compared to AS patients respectively at baseline. Peripheral arthritis, enthesitis, dactylitis, psoriasis, inflammatory bowel disease, hyperlipidemia, cardiac disease, diabetes mellitus, patient global assessment, BASFI, HAQ and SF-36 physical component summary and mental component summary were similar in both groups at baseline. Mean BASDAI, BAS-G, patient global assessment, HAQ and SF-36 physical component summary and mental component summary scores were similar at 12 months between both groups, except for BASFI, which was higher in AS patients $(p<0.001)$. Medications used were similar between AS and $\mathrm{nr}-\mathrm{AxSpA}$ patients $(81 \%$ of axial SpA patients were on NSAIDs, $31 \%$ on concomitant DMARDs and $14 \%$ on biologics). Mean erythrocyte sedimentation rate and C-reactive protein were similar between both groups at baseline and 12 months post treatment.

Conclusions: In our multi-ethnic Asian cohort, patients with AS are more likely to be HLA-B27 positive, have uveitis, hypertension, and poorer physical function despite therapy; whilst $\mathrm{nr}$-axSpA patients have higher baseline disease activity scores and tend to experience poorer well-being and quality of life. Although both groups represent different aspects of the same disease, they respond similarly to treatment.

Disclosure of Interest: None declared

DOI: 10.1136/annrheumdis-2017-eular.3366

\section{SAT0390 DIFFUSION WEIGHTED IMAGING (DWI) IS MORE USEFUL THAN SHORT TAU INVERSION RECOVERY (STIR) SEQUENCE IN DIAGNOSIS OF NON-RADIOGRAPHIC AXIAL SPONDYLOARTHRITIS (SPA) AND BOTH SEQUENCES ARE PARTICULARLY USEFUL IN EARLY DISEASE STAGE}

C.W.S. Chan, H.Y. Chung. Medicine, Queen Mary Hospital, Hong Kong, Hong Kong

Background: Diffusion weighted imaging (DWI) is a new Magnetic Resonance Imaging (MRI) sequence proposed for spondyloarthritis (SpA) diagnosis. Whether it is more useful than the traditional short tau inversion recovery (STIR) sequence in disease diagnosis had not been evaluated.

Objectives: By comparing with traditional STIR sequence in a group of back pain patients newly referred to rheumatology clinics, we evaluated the usefulness of DWI in SpA diagnosis at different stages.

Methods: All new patients referred to the rheumatology clinics with persistant back pain were recruited. DWI and STIR MRI were performed. Conventional radiographs of the pelvis were assessed according to the modified New York criteria for ankylosing spondylitis. Bone marrow edema (BME) and active sacroilitis according to the ASAS definition were evaluated in STIR and DWI by two independent observers.

Results: One hundred and thirty-three patients were recruited. Ninety patients $(67.7 \%)$ had a clinical diagnosis of SpA. Average back pain duration was $8.5 \pm 8.9$ years. The presence of Human Leukocyte Antigen (HLA) B27 was found in $42.9 \%$ of the study population. Inter-observer correlations were excellent (STIR $95.4 \%, p<0.001$; DWI $69.5 \%, p<0001$ ). DWI was found to be comparable to STIR in disease diagnosis (sensitivity DWI $34.1 \%$ vs STIR $34.3 \%$; specificity DWI $85 \%$ vs STIR $93.8 \%$ ) and when applied to the Assessment of SpondyloArthritis international Society (ASAS) criteria for axial SpA (sensitivity DWI $78.9 \%$ vs $79.5 \%$; specificity DWI $75.0 \%$ vs $78.8 \%$ ). DWI is better than STIR in nonradiographic axial SpA group (sensitivity DWI $37.8 \%$ vs STIR $33.8 \%$; specificity DWI $85.3 \%$ vs STIR $95.6 \%$ ). In the group with disease duration less than 3 years, the two images showed improved sensitivity (sensitivity DWI $34.5 \%$ vs STIR $41.4 \%$; specificity DWI $84.6 \%$ vs STIR $88.5 \%$ ).

Conclusions: DWI is useful in $\mathrm{SpA}$ diagnosis especially in non-radiographic axial SpA group. Both DWI and STIR are especially useful for early disease diagnosis. Disclosure of Interest: None declared

DOI: 10.1136/annrheumdis-2017-eular.2173

\section{SAT0391 LUMBAR FLEXION/RELAXATION PHENOMENON IN PATIENTS WITH AXIAL SPONDYLOARTHRITIS}

C. Aranda-Valera ${ }^{1}$, J.L. Garrido-Castro ${ }^{2}$, A.M. Galisteo ${ }^{1}$, J. Peña-Amaro ${ }^{1}$ C. González-Navas ${ }^{2}$, P. Font-Ugalde ${ }^{2}$, E. Collantes-Estevez ${ }^{2} .{ }^{1}$ University of Cordoba; ${ }^{2}$ Maimonides Institute for Biomedical Research of Cordoba, Cordoba, Spain

Background: Surface electromyography (sEMG) has been used in several studies to assess muscle activity in patients with low back pain (LBP). It has also been analyzed the lumbar flexion-relaxation phenomenon (FRP) defined by reduced lumbar erector spinae $(E S)$ muscle myoelectric activity during full trunk flexion in healthy individuals, through the flexion relaxation ratio (FRR). In LBP patients, this relaxation, compared to the peak reached at the flexion phase, is smaller and even non-existent. There are very few studies that analyze this effect in patients with Axial Spondyloarthritis (axSpA).

Objectives: To evaluate muscular activity at the erector spinae using sEMG in patients with axSpA to analyze FRP.

Methods: 39 subjects were included: 25 patients with axSpA $(49.3 \pm 5.6$ years, $75 \%$ men) and 14 healthy subjects ( $46.7 \pm 8.7$ years, $71 \%$ men) as control group. Demographic data, conventional metrology, advanced metrology using motion capture (UCOTrack) and PRO questionnaires were collected. Electrodes were placed on left and right side, at L4-L5 level and separated $2 \mathrm{~cm}$ from the spinous process, on the ES. Muscle activity values were obtained in 4 phases (standing, flexion, relaxation and extension). With the values of flexion and relaxation, the FRR index and its inverse 1/FRR were calculated. Student t tests were used for differences between groups.

Results: There were no significant differences between the right and left sides of the measurements at ES, so mean values were considered for the analysis. There were also no significant differences in age and gender between the control group and patients. Results obtained in each of the phases are shown in the graph. The FRP appeared in healthy individuals, but not in patients, as show the FRP line in the graph: in axSpA, sEMG values at the Flexion phase are above values at the stand phase, and in control group is the opposite, so there is a truelly relaxation. There were significant differences between patients and control group in flexion, relaxation, and in the FRR and 1/FRR ratios. 1/FRR presented better correlations with several parameters (age, lateral flexion, Schöber, BASMI, BASFI, UCOASMI, BASG, all with $p<0.01$ ), but not with activity indexes (BASDAI, ASDAS). Distribution of 1/FRR values and results of $S E M G$ between group $\left({ }^{* *} \mathrm{p}<0.01,{ }^{*} \mathrm{p}<0.05\right)$ are shown at the chart. The area under a ROC curve to discriminate healthy patients using 1/FRR was $0.85(95 \% \mathrm{Cl}$ $0.72-0.96 \mathrm{p}<0.001)$.
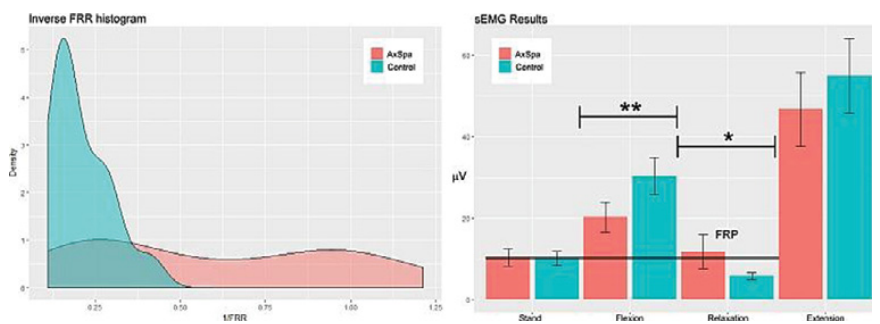

Conclusions: In our study, there were differences between healthy and patients with axSpA in the FRP, as in other studies with LBP patients. The 1/FRR index shown the best results in correlation with other parameters and it was where major differences between groups appeared. There was good correlation with the patient global score (BASG) and with the functional BASFI index, so assessment with sEMG could be an objective and quantitative test to evaluate the patient's functional status. It would be very interesting to analyze, in future studies, the sensitivity to change to treatments which would give us a good indicator to assess their effectiveness.

Acknowledgements: This study was supported by the XXI Research Promotion Program of the University of Córdoba (Spain)

Disclosure of Interest: None declared

DOI: 10.1136/annrheumdis-2017-eular.2575

\section{SAT0392 THE DIAGNOSTIC ACCURACY OF EXISTING GRADING CRITERIA OF SACROILIAC JOINT CT IN ANKYLOSING SPONDYLITIS}

D. Gao, K. Li, Y. Wang, J. Zhu, J. Zhang, F. Huang. Rheumatology, Chinese PLA General Hospital, Beijing, China

Background: Imaging modalities are essential for the diagnosis of ankylosing spondylitis (AS) due to the absence of specific clinical manifestations. Sacroiliac Joint (SIJ) CT has been used to identify sacroiliitis for decades with a higher diagnostic accuracy than radiography in detecting structural changes, and not reducing the specificity like MRI. However, no well-accepted grading system for SIJ CT existed

Objectives: We evaluated the diagnostic accuracy of existing grading criteria of sacroiliitis, aiming to provide references for future better reading of SIJ CT in AS. Methods: A total of 2714 patients who had received CT scanning for any reasons with complete SIJ structures displaying between June 2012 and December 2015 were included. The CT scans were read by 2 rheumatologists together who had received professional training in radiology. Patients with sacroiliitis were selected and bilateral SIJs of each patient were evaluated separately by the 1984 modified New York (mNY) criteria, the criteria proposed by Lee (Lee criteria) ${ }^{[1]}$ and the criteria from Innsbruck workshop report (Innsbruck criteria) ${ }^{[2]}$, respectively. The grading differences among these criteria were analyzed.

Results: Amount to 509 patients were detected having sacroiliitis with an average age of 34 years and $64 \%$ of male. Among 1018 SIJs of these patients, 45 SIJs graded $1 \sim 3$ by $\mathrm{mNY}$ criteria were graded 0 by Lee criteria, indicating the better specificity of Lee criteria. Lee criteria was much more convenient and reliable than $\mathrm{mNY}$ criteria for its more explicit definitions. The SIJs with definite sacroiliitis estimated by $\mathrm{mNY}$ and Lee criteria were $79.37 \%$ and $82.91 \%$, respectively, and simply divided into grade 3 or grade 4 . Conversely, $85.27 \%$ SIJs were identified as definite sacroiliitis and classified into 5 grades, from grade IIB to grade IVB, by Innsbruck criteria, and the percentages of each grade were $8.94 \%, 26.82 \%$, $20.92 \%, 10.12 \%$ and $18.47 \%$, respectively, which means a higher discrimination capability than the other two criteria. Other than graded by the extent of lesions in $\mathrm{mNY}$ criteria or Lee criteria, the grading assessment by Innsbruck criteria was based on the lesion types, which was more consistent with the natural progression of sacroiliitis. 
Abstract SAT0392 - Table 1. The existing grading criteria of SIJ CT

\begin{tabular}{|c|c|c|}
\hline mNY criteria & Lee criteria & Innsbruck criteria \\
\hline $\begin{array}{l}0=\text { Normal } \\
1=\text { Suspicious for erosions or sclerosis } \\
2=\text { Mildly abnormal with definite erosions or sclerosis, but without alteration in the joint width } \\
3=\text { Moderately abnormal with erosions or sclerosis, joint space narrowing or widening } \\
\text { and/or partial ankylosis } \\
4=\text { Complete ankylosis }\end{array}$ & $\begin{array}{l}0=\text { Normal } \\
1=\text { Focal erosions seen on only one of either semi-coronal or axial } \\
\text { images } \\
2=\leq 25 \% \text { erosions }{ }^{\star}, \text { but without alteration in the joint width } \\
3=\geq 25 \% \text { erosions }{ }^{\star} \text {, joint space alteration and/or partial ankylosis } \\
4=\text { Complete ankylosis }\end{array}$ & $\begin{array}{l}\mathrm{IA}=\mathrm{SIJ}>4 \mathrm{~mm} \\
\mathrm{IB}=\mathrm{SIJ}<2 \mathrm{~mm} \\
\mathrm{IIA}=\text { Contour irregularities } \\
\mathrm{IIB}=\text { Erosion } \\
\text { IIIA }=\text { Subchondral sclerosis } \\
\text { IIIB }=\text { Spur formation } \\
\text { IVA }=\text { Transarticular bony bridges } \\
\text { IVB }=\text { Ankylosis }\end{array}$ \\
\hline
\end{tabular}

${ }^{*}$ Extent of erosions; $(\%)=$ number of slices with erosions/total number of slices $\times 100$.

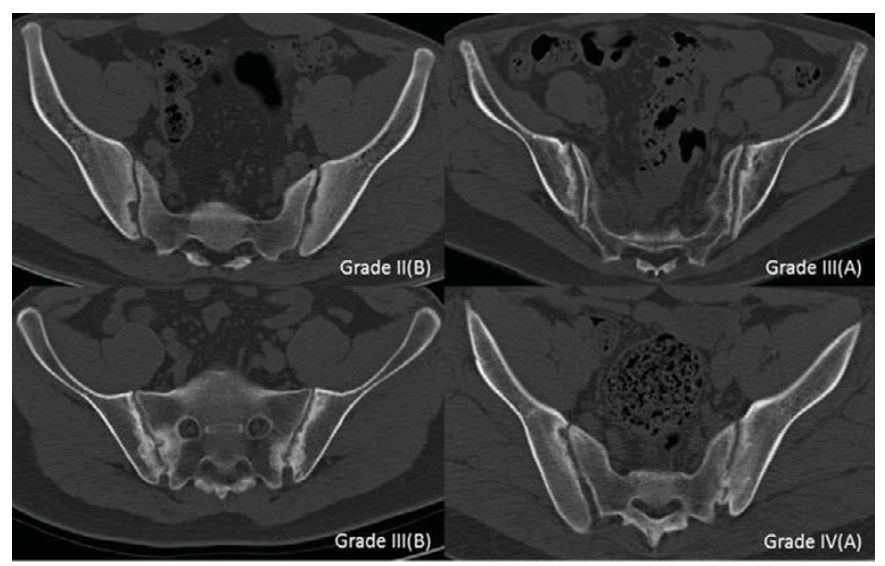

Figure 1 SIJ CT images graded 3 by $\mathrm{mNY}$ criteria or Lee criteria, while graded from II(B) to IV(A), respectively, by Innsbruck criteria.

Conclusions: Lee criteria has a better diagnostic specificity with a lower difficulty in the evaluation process, while Innsbruck criteria is a more detailed grading system, which has a higher consistency with the progression of sacroiliitis in AS. References:

[1] Lee YH, et al. Rheumatol Int, 2013, 33(4): 1005-1011.

[2] Klauser A, et al. J Rheumatol 2004, 31(10): 2041-2047.

Disclosure of Interest: None declared

DOI: 10.1136/annrheumdis-2017-eular.4224

\section{SAT0393 PROTEIN FINGERPRINTING SCREENING SPECIFIC PROTEINS IN SERUM OF PATIENTS WITH ANKYLOSING SPONDYLITIS}

\section{Ma, L. Zhang. Rheumatology Department, Shanxi DaYi Hospital, Shanxi} Academy of Medical Sciences, Taiyuan, China

Background: Ankylosing spondylitis (AS) is a chronic inflammatory rheumatic diseases which mainly affects the spine and sacroiliac joint. So far, the pathogenesis of AS remains elusive, making it difficult to improve early diagnosis and treatment. Proteomics is a new enabling technology to screen disease associated proteins which can be used in diagnostics or therapeutics.

Objectives:

The surface-enhanced laser desorption ionization/time of flight mass spectrometry (SELDI-TOF-MS) and protein chip screening specific biomarkers in serum of patients with ankylosing spondylitis (AS) are used to diagnose and assess the disease as well as to anticipate the program of disease.

\section{Methods:}

The serum samples of 69 AS patients, 10 rheumatoid arthritis (RA) and 12 healthy individuals were detected by SELDI-TOF-MS and weak cation exchange (WCX-2) chip. Then 69 AS patients were divided into several types such as the active and inactive stage of illness, axial arthritis involved and peripheral and axial arthritis involved, the positive and negative group of HLA-B27 to study differentially expressed proteins in the pathogenesis of AS by using Biomarker Wizard and Biomarker Pattern software of SELDI to screen the specific proteins and set up the diagnostic prediction models of disease.

Results: 1 . The contents of 27 proteins between AS patients and healthy groups were significantly different. Of these proteins, 14 were up-regulated and 13 were down-regulated in patients with AS. The diagnostic model made up of 8085, 2640 and 2932 could be used to diagnose AS, which the sensitivity and specificity were $94.23 \%$ and $100 \%$ respectively.

2.The contents of 30 proteins were significantly different. Of these proteins, 14 were up-regulated and 16 were down-regulated in the active stage of AS. The diagnostic model made up of $3677,3880,2539,3159$ and 3242 could be used to determine the disease activity of AS, which the sensitivity and specificity were $98.11 \%$ and $100 \%$ respectively.

3. The contents of 3 proteins were significantly different. The protein of $M / Z 8687$ was up-regulated in the axial arthritis involved of $A S$, while the proteins of $M / Z$ 4700,18538 were down-regulated. The diagnostic model made up of the three proteins could be used to predict AS whether peripheral arthritis was involved or not, which the sensitivity and specificity were $80.00 \%$ and $82.35 \%$ respectively.
4.There were no different expressed proteins in serum between the positive and negative group of HLA-B27.

5.The contents of 23 proteins were significantly different. Of these proteins, 14 were up-regulated and 9 were down-regulated in the AS patient. The diagnostic model made up of 10259, 7972, 2048, 2154 and 2954 could be used to distinguish AS and RA, which the sensitivity and specificity were $100 \%$ and $100 \%$ respectively.

\section{Conclusions:}

The serum protein fingerprinting set up by SELDI-TOF-MS could screen new biomarkers in AS, which is expected to become a screening platform in diagnose and assessment of disease.

\section{References:}

[1] Kabeerdoss J, Kurien BT, Ganapati A, et al. Proteomics in rheumatology[J]. Int J Rheum Dis, 2015, 18(8): 815-817.

[2] Li Y, Sun $X$, Zhang $X$, et al. Establishment of a decision tree model for diagnosis of early rheumatoid arthritis by proteomic finger printing[J]. Int $\mathrm{J}$ Rheum Dis, 2015, 18(8): 835-841.

[3] Liu J, Zhu P, Peng J, et al. Identification of disease-associated proteins by proteomic approach in ankylosing spondylitis[J]. Biochem Biophys Res Commun, 2007, 357(2): 531-536.

Disclosure of Interest: None declared

DOI: 10.1136/annrheumdis-2017-eular.3342

\section{SAT0394 THE IMPAIRMENT OF HIP JOINTS IN PATIENTS WITH EARLY AXIAL SPONDYLOARTHRITIS (CORSAR COHORT) BASED ON THE RESULTS OF THE TWO-YEAR OBSERVATION}

D. Rumiantceva ${ }^{1}$, T. Dubinina $^{1}$, O. Rumyantseva ${ }^{1}$, A. Demina ${ }^{1}, E_{\text {. Agafonova }}{ }^{1}$ S. Erdes ${ }^{1}$, S. Krasnenko ${ }^{1}$, A. Smirnov ${ }^{2} .{ }^{1}$ Laboratory of spondyloarthritis;

${ }^{2}$ Laboratory of radiology, Nasonova Research Institute of Rheumatology,

Moscow, Russia, Moscow, Russian Federation

Background: Previous studies showed that the impairment of hip joints $(\mathrm{HJ})$ coxitis leads to a hip replacement and it is a frequent cause of early disability in patients (pts) with spondyloarthritis $(\mathrm{SpA})$. Early detection of coxitis have a great clinical importance.

Objectives: To study the incidence and character of the impairment of $\mathrm{HJ}$ involvement in patients with early axial $\mathrm{SpA}$ (axSpA).

Methods: The study include 65 patients with axSpA (ASAS 2009) with disease duration $<5$ years and age at onset $<45$ years, mean age $28,5(5,8)$ y., $32(49,2 \%)$ males, $60(92,2 \%)$ pts were HLAB27-positive, average disease duration was 24, $(15,4)$ mo. The following evaluations were made: HJ pain (numerical rating scale - NRS - from 0 to 10), inter-malleolar distance (IMD), radiological HJ changes (BASRI-hip), ultrasound examination (US) and pts who had US evidence or/and clinical coxitis - MRI of hip joints. For 2 years pts taking NSAIDs at therapeutic doses. The dosages of NSAIDs accounted by the ASAS NSAID index.

Results: After 2 years follow-up reduce $\mathrm{HJ}$ functional limitations and pain in the

Table 1. Clinical characteristics of imparment $\mathrm{HJ}$ at baseline and after 2 years

\begin{tabular}{lccc}
\hline & Baseline & After 2 years & P \\
\hline Bilateral HJ pain, \% pts & $22(33,8 \%)$ & $17(26,1 \%)$ & 0,2 \\
Pain in the right HJ, \% pts & $6(9,2 \%)$ & $5(7,6 \%)$ & 0,5 \\
Pain in the left HJ, \% pts & $32(49,2 \%)$ & $8(12,3 \%)$ & 0,000003 \\
HJ funcional limitations, \% pts & $13(20,0 \%)$ & $3(4,6 \%)$ & 0,007 \\
Bilateral HJ funcional limitations, \% pts & $8(12,3 \%)$ & $3(4,6 \%)$ & 0,1 \\
IMD, mean (s.d.) & $110,8(11,0)$ & $110,8(11,0)$ & 1,0 \\
\hline
\end{tabular}

Table 2. MRI symptoms of the impairment HJ and US symptoms of coxitis at baseline and after 2 years

\begin{tabular}{lccc}
\hline & Baseline & After 2 years & P \\
\hline MRI symptoms of the defeat HJ, \% pts & $22(68,7 \%)$ & $14(43,7 \%)$ & 0,08 \\
Bilateral synovitis, \% pts & $17(77,2 \%)$ & $10(71,4 \%)$ & 0,5 \\
One-side synovitis, \% pts & $4(18,1 \%)$ & $2(14,2 \%)$ & 0,5 \\
Bilateral swelling of bone marrow in femoral head, \% pts & $1(4,5 \%)$ & 0 & 0,5 \\
One-side swelling of bone marrow in femoral head, \% pts & $2(9,0 \%)$ & 0 & 0,2 \\
Bilateral swelling of acetabular roof, \% pts & $2(9,0 \%)$ & $3(21,4 \%)$ & 0,3 \\
One-side swelling of acetabular roof, \% pts & 0 & $1(7,1 \%)$ & 0,5 \\
US symptoms* of coxitis, \% pts & $14(21,5 \%)$ & $11(16,9 \%)$ & 0,3 \\
Bilateral US symptoms of coxitis, \% pts & $2(14,2 \%)$ & $4(28,5 \%)$ & 0,3 \\
One-side US symptoms of coxitis, \% pts & $12(85,7 \%)$ & $7(50,0 \%)$ & 0,9
\end{tabular}

$\begin{array}{llll}\text { One-side US symptoms of coxitis, \% pts } & 12(85,7 \%) & 7(50,0 \%) & 0,9\end{array}$

*The distance between the anterior joint capsule and the femoral neck, capsular-neck distance CND $>7 \mathrm{~mm}$. 